\title{
Metabolic screening in drug development: in-vivo to in-silico
}

\begin{abstract}
Inter-individual differences in drug action and side-effects have given rise to various types of metabolic screening techniques in drug development. They range from in-vitro, invivo techniques to sophisticated in-silico techniques. While validation of most systems is still largely pending, such techniques offer predicting various characteristics of drugs, namely metabolic stability, consequent half-life, dosage schedule, or induction potential. The techniques are getting refined everyday which offers better avenues of research and drug development. We discuss in brief latest metabolic screening techniques currently in use.
\end{abstract}

Keywords: drug metabolism, metabolic stability, drug interactions, high-throughput screens
Volume 6 Issue 4 - 2017

\author{
Roy Ritam, Lahiry Sandeep \\ Department of Pharmacology, Institute of Post Graduate
}

Medical Education and Research, India

Correspondence: Sandeep Lahiry, Department of Pharmacology, Institute of Post Graduate Medical Education and Research, 244 B A.J.C Bose Road, Kolkata, India, Email sndplry@gmail.com

Received: October 23, 2017| Published: December 08, 2017
Abbreviations: ADME, absorption, distribution, metabolism and excretion; NDA, new drug application; FDA, food and drug administration; CYP, cytochrome P; UGT, uridine diphosphoglucuronosyl transferase; NAT, N-acetyl transferase; GST, glutathione s-transferase; ST, sulfo transferase; QSAR, quantitative structureactivity relationships; PCA, principal components analysis; CoMFA, comparative molecular field analysis; BBB, blood-brain barrier; COMPACT, computer-optimised molecular parametric analysis of chemical toxicity

\section{Introduction}

New drug development is very laborious and long-term process. It needs so much effort, time and resources that failure to get proper therapeutic drug from a possible candidate is unacceptable commercially in this competitive market. A successful drug candidate needs to be efficacious without unacceptable adverse effects. There are thousands of probable drug candidates are present but a very few can be able to be used as drugs because of its effects to the body with adverse effects. So, prediction of metabolism of the xenobiotic candidate, it's probable adverse effects and risk to benefit ratio are important considerations for a new drug development process. Historically, we use In vivo technique in which new drug is administered into experimental animals to observe its effects and most importantly its toxicity. In-Vitro methods are developed in which drugs are tested in laboratory environments in properly maintained tissues or part of the organs. But thousands of thousands of data being generated worldwide due to advent of different branches of science e.g. biochemistry, genetics, molecular biology etc along with the field of pharmacology and pharmaceutical researches in last two decade which is well beyond the capacity of 'in-cerebro'. So, to get benefit from modern developing sciences in drug developments we need a new tool that is based on computer or 'in-silico' techniques. As computer based systems can hold big enough databases in one place and calculate hundred times faster than human capacity, time consuming new drug development process can be benefited from it. ${ }^{1}$

\section{Drug discovery and development pathway}

Drug discovery and development beginning with target identification and validation. Target can be DNA, RNA or any protein that contributes for the disease. Its validation done by demonstrating that modulating the target has a therapeutic effect. Next step is to find compounds that actively engage the target. From the many potential compounds, a few leads that show a relationship between chemical structure and target-based activity in a biochemical or cellbased assay are identified. Target assay may include complex cellular assays, toxicological assays, bio-pharmacological assays, and assay for absorption, distribution, metabolism, and excretion (ADME) profile. $^{2}$

Animal models (in-vivo) are useful for prioritising potential candidates among many all that can be chosen for clinical trial. Along with this in-vitro techniques are done. By In-vitro technique several biochemical tests are done in lab to detect major metabolic pathway of the molecule. All these data obtained from in-vivo and invitro procedures are needed to establish 'Proof of Concept'. Then Phases of human testing (Clinical Trials) are done to ensure safety and efficacy over human subjects. After successful completion of Phase III clinical trial and submission of a new drug application (NDA) to the U.S. Food and Drug Administration (FDA), a drug becomes eligible for marketing. Even after marketing a drug continues to be studied through post-marketing surveillance to ensure safety. ${ }^{3}$

\section{In vivo methods, pros and cons}

In vivo methods or Animal models are used to know whether the new molecule is pharmacologically inert or functional in modifying the target. If the drug is actually acting then what are its effects, adverse toxic, dose dependency, effects on deferent organ systems, distribution, excretion data etc. Animal model tends to give many useful information, but the major disadvantage is animal physiology may not reflect and behave as human physiology. Therefore, the molecules may act differently in animals and pharmacokineticpharmacodynamic profile may be different. But In-vivo methods are useful in prioritising different possible pharmacologically active compounds as the drug development procedure progresses. ${ }^{4}$

\section{In-Vitro Methods}

Although many other organs involved in drug biotransformation, but as liver is the most important among all, so an in-vitro model usually focuses on tissues or cells obtaining from liver. There are several advantages as well as disadvantages of In-Vitro models. But still in-vitro models are one of the most popular ways of metabolic screening of new chemical compounds. 
Drug biotransformation is divided into two types of reactions, namely phase I (hydrolysis, oxidation, and reduction) and phase II reactions (conjugation). The biotransformation pathway of a drug is mediated by phase I, phase II, or a combination of both. The cytochrome P-450 (CYP) enzyme superfamily plays a dominating role in the phase I biotransformation which is mainly present in liver. Phase II enzymes (e.g., uridine diphospho-glucuronosyl transferase (UGT), N-acetyl transferase (NAT), glutathione S-transferase (GST), and sulfotransferase (ST)) also have an important role in the detoxification and/or excretion rate of drugs.

Liver tissues (Microsome, supersomes or hepatic tissues as a whole) which contain different enzymes in them can be useful in testing and understanding the new molecule's major metabolic pathway and different intermediates that can either influence its effects or can cause adverse effects. Major advantage of this system is reduced complexity of the study system and we can predict preclinically in laboratory what is the new drugs metabolism and its effects on cellular biochemistry. ${ }^{4}$ In vitro screening assays with human liver-derived experimental systems represent the most effective approach to estimate human in vivo drug metabolic fates. This system contains followings,

\section{Human liver microsomes}

Microsomes are vesicles that are actually endoplasmic reticulum prepared by homogenization of the liver, followed by a relative low speed centrifugation (e.g. $9000 \mathrm{x} \mathrm{g}$ ) to remove whole cells, free nuclei, plasma membranes and mitochondria. The resulting supernatant, conventionally called S-9, which is sometimes used for metabolism studies, is then centrifuged at a higher speed $(100,000 \mathrm{x} \mathrm{g})$, to pellet the endoplasmic reticulum, which contains the P-450 isoforms and one of the phase II conjugating enzymes, UGT.

\section{Human hepatocytes}

Human hepatocytes contain virtually all metabolic enzymes. They can be obtained from culturing or cryo-preservation of human hepatocytes or by obtaining liver slices or part of the liver itself. Human liver microsome and S-9 Fraction are two most popular models of In-Vitro metabolic screening methods. Although several methods are developed each have their own pros and cons.

\section{Major advantages}

In-Vitro methods are easy way of performing and predicting major metabolic reactions in laboratory. They can be done in relatively low cost. We also can automate the process by using machines or tools. It can simulate and confirm In-Vivo test data that are already been documented. With added convenience In-Vitro models are commercially available and can be stored in cryopreservation or by other means.

\section{Major disadvantages}

Although In-Vitro methods are very helpful in drug development, but there are some major disadvantages also. Results obtained with microsomes cannot be used for quantitative estimations of in vivo human biotransformation, because CYPs and UGTs are enriched in the microsomal fraction and there is no competition with other enzymes. This result in higher biotransformation rates in microsomes compared to the human In-Vivo situation. In liver microsomal model, the absence of different enzymes and cytosolic cofactors can leave metabolites formed in intact liver cells unnoticed. Human S-9 fractions offer more complete metabolic screening than other methods but its major disadvantage is overall lower enzyme activity and leave some metabolites unnoticed. In isolated hepatocyte method, lack of non-hepatocytic cells (e.g. Kupffer's cell) limits co-factor supply and thus limits overall exploration. Availability of viable hepatic tissues including human hepatic slices or infused organ are also a major challenge in many laboratories. Moreover, there is considerable inter personal variations in different In-Vitro models such as in human microsome and hepatocyte model. ${ }^{4}$

\section{In-silico methods}

With the increasing rapid search for new molecules in pharmaceutical industry, agrochemical products and personal products thousands of new probable effective molecules are separated and analysed every year. Now to test, analyse and predict ADME profile of this huge number of new molecules in short time emergence of computer technology based methods are inevitable. This computer based systems are known as In-Silico methods. ${ }^{5}$

\section{Quantitative structure-activity relationships (QSAR)}

In-Silico model is actually based on Quantitative StructureActivity Relationships (QSAR). The concept of QSAR is there is a persistent relationship between chemical structures with its biological activity. So in In-Silico methods, computerised database and algorithm assess the molecules based on its chemical structures both two and three dimensionally. Much of the work in this field is focused on substrate, inhibitors and inducers of various isoenzymes of Cytochrome P-450 system. Inherent metabolic stability has also has been modelled using principal components analysis (PCA) on large corporate databases. As mammalian Cytochrome P-450 enzymes are membrane bound, so the crystal structures of these enzymes are not available. Mammalian cytochrome homology model have been built incorporating a knowledge of prokaryote cytochrome structures. Relating the findings of substrate docking experiments vastly expands the knowledge of classic QSAR model in xenobiotic research. Techniques such as comparative molecular field analysis (CoMFA) and substrate pharmacophore modelling have enabled interpretation and understanding of enzyme active sites even when a crystal structure or homology model is not available. ${ }^{6}$

Two major types of In-Silico systems are available. First, enzyme modelling systems based on theoretical and mechanistic considerations and second, empirically based expert systems which rely on expert rules as the basis of their predictions. A third category also exists that contain classical reaction databases.

\section{Enzyme modelling methods}

COMPACT (Computer-Optimised Molecular Parametric Analysis of Chemical Toxicity): COMPACT system assesses the ability of the new molecule to form enzyme-substrate complex by the CYP1A and CYP2E subfamilies of cytochrome P450s. This system has been developed at University of Surrey, Guildford, UK by Lewis and coworkers.

Camitro: Camitro Corporation suite of ADME model includes modules for cytochromeP450 metabolism, blood-brain barrier (BBB) partitioning, human intestinal absorption and aqueous solubility. Camitro's models are based on a novel, combined empirical and quantum chemical approach to predict enzyme-substrate binding affinities metabolic sites, and relative rates of metabolism at discrete sites within a molecule. Three major cytochrome P450 enzymes are focused CYP3A4, CYP2D6, and CYP2C9, together they contribute to metabolism $90 \%$ of clinically used drugs. 


\section{Expert systems}

META: This system is capable of predicting possible sites of enzymatic attacks and nature of chemicals formed by such biotransformation. It uses database of biotransformation which was created by experts in the field of xenobiotic metabolism. It predicts the biotransformation by detecting key functional groups within complete chemical structures. After each biotransformation, a stability check is performed on the reaction product. A simple quantum mechanical calculation detects unstable atom arrangements and program transforms products containing them into more stable arrangements. This process continues until a stable arrangement is calculated. Thus, this programme can then evaluate stable metabolites and attempt to transform them to build a complete metabolic tree.

Metabol expert: This system is composed of one or more databases, a knowledge base and one or more prediction tools. The transformation knowledge base is composed of 'if...., then...' Type rules each of biotransformation rule is composed of four components: Substructure changed during transformation, new substructure formed, a list of substructures one of which must be present in the molecule for the biotransformation to occur and a list of substructures whose presence prevents the transformation from occurring. This system uses a graphical means of structure input and the results are also displayed graphically. System is interactive and users can modify and rearrange metabolic tree graphically.

METEOR: It is a computer system which uses a knowledge-base of structure-metabolism rules to predict the metabolic fate of a chemical structure. This system is made by LHASA Limited, Leeds, UK.

\section{Database systems}

MDLI Metabolite database: It is metabolism information system. It comprises a database, a registration system and a browsing interface. It uses information from different studies to assemble structural metabolic database entries for particular parent compounds. It covers literature from year 1901 to present day. The prime focus is on xenobiotic compounds and biotransformation of medicinal drugs.

Accelrys' Biotransformations database: Developed in conjunction with the Royal Society of Chemistry, Accelrys' Biotransformations databaseprovides structure-searchable access to this well-respected reference work on a single, easy to use CD-ROM.

\section{Conclusion}

In this increasingly competitive market, failure to get approval for a new molecule as drug is a big issue as to develop, research and market a new molecule as drug needs considerable amount of resources and efforts of the concerned people and sponsors. Therefore, newer technology and knowledge-based methods are important in drug development field to make the process easier and less time consumable. In-Vivo and In-Silico methods are established and reliable tools of metabolic screening for past several decades. But with increasing discovery of target modifying molecules and vast amount of collaborative knowledge worldwide industry now needs a reliable computer based model to maintain all the records as well as analyse new molecules with ease. So, emerging In-Silico methods are already makes preclinical metabolic screening process simpler. It is constantly updated and will probably establish itself as a reliable tool for drug development process in coming years.

\section{Acknowledgements}

None.

\section{Conflicts of interest}

Author declares there are no conflicts of interest.

\section{Funding}

None.

\section{References}

1. Robinson SW, Fernandes M, Husi H. Current advances in systems and integrative biology. Computational and Structural Biotechnology Journal. 2014;11(18):35-46.

2. https://www.ncbi.nlm.nih.gov/books/NBK195047/

3. Umscheid CA, Margolis DJ, Grossman CE. Key Concepts of Clinical Trials: A Narrative Review. Postgrad Med. 2011;123(5):194-204.

4. https://www.ncbi.nlm.nih.gov/books/NBK100199/

5. Sacan A, Ekins S, Kortagere S. Applications and limitations of in silico models in drug discovery. Methods Mol Biol . 2012;910:87-124.

6. Perkins R, Fang H, Tong W, et al. Quantitative structure-activity relationship methods: perspectives on drug discovery and toxicology. Environ Toxicol Chem. 2003;22(8):1666-1679. 